\title{
Acute presentation of papillary glioneuronal tumor due to intra-tumoral hemorrhage in a toddler: An odd presentation of a rare pathology
}

\section{Amin Tavallaii ( $\nabla$ tavallaeia@mums.ac.ir)}

Mashhad University of Medical Sciences https://orcid.org/0000-0003-2486-2919

\section{Ehsan Keykhosravi}

Mashhad University of Medical Sciences

Hamid Rezaee

Mashhad University of Medical Sciences

\section{Case Report}

Keywords: Focal neurological deficit, Hemorrhage, Papillary glioneuronal tumor, Pediatric, Toddler

Posted Date: May 7th, 2020

DOI: https://doi.org/10.21203/rs.3.rs-27119/v1

License: (1) This work is licensed under a Creative Commons Attribution 4.0 International License.

Read Full License

Version of Record: A version of this preprint was published at British Journal of Neurosurgery on August 12th, 2020. See the published version at https://doi.org/10.1080/02688697.2020.1800588. 


\section{Abstract}

Background and Importance: Papillary glioneuronal tumor is a recently known entity in central nervous system tumors. These benign WHO grade I tumors are mostly seen in young adults. Pediatric PGNT is rare and there is no report of these tumors in toddlers. Headache, nausea/vomiting and seizure are most common clinical symptoms. Acute presentation with focal neurological deficits or loss of consciousness are not amongst the expected presentations. These tumors are typically cystic with enhancing mural nodule. Although case with chronic intermittent microhemorrhages are reported in the literature but overt intra-tumoral hemorrhage is an odd radiological presentation with just one reported case in the literature.

Clinical presentation: We present an extremely rare case of PGNT presenting with sudden onset hemiparesis and impaired consciousness due to acute intra-tumoral hemorrhage in a toddler which was surgically treated with favorable outcome.

Conclusion: PGNTs can also be seen in very young children even in toddlers. Also, it should be kept in mind that these tumors have potential for overt intra-tumoral hemorrhage and acute presentation with focal neurological deficits mimicking more common pathologies which should be considered to plan optimal patient management.

\section{Background And Importance}

Papillary glioneuronal tumor (PGNT) is a rare brain tumor which is recently recognized as a new entity by the updated 2007 world health organization (WHO) classification of tumors of central nervous system (CNS)[27]. Although this tumor was first described by Komori et al in 1998[23], before being known as a separate entity it was considered as a variant of ganglioglioma in 2000 WHO classification[22]. These tumors tend to occur more commonly in young adults and are mostly seen in deep paraventricular areas of cerebral hemispheres with tendency to temporal lobe[23,42,37,44]. Only 150 cases of PGNTs are reported up to now in the literature[36,31,17,11,41,15,12,2]. Amongst these, only 41 cases are reported in pediatric population which demonstrates rarity of this pathology in this age group $[11,43,44,32,35,29,26,8,1,42,30,33,28,34,20,18,19,14,16,3,39,13,10,38,7,25,9,4,23]$. To our knowledge, there is no report of PGNT in a toddler up to now. These slow growing tumors are mostly diagnosed after occurrence of slowly progressive raised intracranial pressure symptoms such as headache, nausea/vomiting or seizure (90.1\%)[37] and acute symptomatology or focal neurological deficits are not amongst reported natural course of these tumors. On the other hand, PGNTs are solid, cystic or more commonly solid-cystic tumors with or without an enhancing mural nodule. Although calcifications can be seen within tumor, intra-tumoral hemorrhage is an extremely rare radiological presentation of these tumors[6,42]. Hemorrhage in form of chronic microhemorrhages leading to hemosiderosis is rarely reported $[43,5]$ but we believe that this is the second report of an acute intra-tumoral hemorrhage in these tumors. So, here we present the first report of PGNT in a toddler with extremely rare acute hemorrhagic presentation leading to focal neurological deficit which was diagnosed and surgically treated with favorable outcomes. 


\section{Clinical Presentation}

History and physical examination:

A two years old male toddler was transferred to our center from a small town in the province with history

of sudden onset left hemiparesis and lowering of consciousness since morning of admission day. There were no history of seizure attack or trauma. Patient was admitted to our center with Glasgow Coma Scale (GCS) of 13, agitated and without appropriate cooperation. Vital signs were within normal range and no abnormality was seen in initial laboratory testing which was performed in referring hospital. Physical examination revealed left hemiparesis with muscle forces of 2/5 and 3/5 in left upper and lower limbs respectively in the Medical Research Council (MRC) scale. Muscle spasticity or hyperreflexia was not present but left plantar reflex was upward. All cranial nerves examinations were intact as far as patient cooperation allowed. Head circumference was within normal range. Fundoscopic examination was not possible and not performed due to patient agitation. Because, there were no history of seizure and patient hemiparesis did not improve over time, a possibility of post ictal tod's paralysis was unlikely.

\section{Imaging findings:}

Patient was brought to us with a non-contrast CT scanning which showed a relatively large, round and well-defined lesion adjacent to frontal horn of right lateral ventricle and head of right caudate nucleus extending superiorly to centrum semiovale and anteriorly. Tumor was significantly compressing and displacing left frontal horn inferiorly and medially and midline shift of almost $1 \mathrm{~cm}$ was also evident. Lesion had a heterogenous density with a hypodense compartment relative to brain parenchyma but hyperdense relative to CSF in favor of cystic lesion and a hyperdense part demonstrating acute hemorrhage. Peripheral segments of lesion were hyperdense in some parts and iso to hypodense in other parts. Significant vasogenic edema was also evident in the adjacent brain parenchyma. There was no sign of hydrocephalus (Figure 1 A-C).

Because of unavailability of emergent MRI at the time and the need to evaluate lesion enhancement and rule out potential vascular lesions, we performed an emergent contrast enhanced CT/CTA. Lesion did not show any vascular connections to the adjacent vasculature such as anterior cerebral artery or smaller branches nearby which somewhat relieved our concerns about vascular anomalies. Lesion showed a significant rim enhancement which was demonstrating enhancing wall of the cyst (Figure 1 D-F).

\section{Management and surgical technique:}

Given the clinical situation of patient, an urgent surgical management was performed. After administration of total intravenous general anesthesia, patient was positioned in neutral supine position with his head placed on a ring head holder and slight neck flexion with attention paid to not compromise venous drainage. Potential pressure points were carefully padded and warming blanket was used to avoid hypothermia during surgery (Figure $2 \mathrm{~A}, \mathrm{~B}$ ). 
After administration of prophylactic antibiotic (Cefalexin) and prophylactic antiepileptic (Phenytoin), a horse-shoe incision was made on right frontal region extending medially to midline and posteriorly to coronal suture. After subgaleal elevation of skin flap, a rectangular craniotomy was performed extending from $1 \mathrm{~cm}$ anterior to coronal suture $3 \mathrm{~cm}$ anteriorly and from approximately $1 \mathrm{~cm}$ off midline $2 \mathrm{~cm}$ laterally (Figure 2C). A U-shaped dural incision was made and dura was reflected medially. After identification of middle frontal gyrus, a small corticotomy and subsequent blunt dissection was performed through white matter fibers with a posterior, inferior and medial trajectory to reach tumor. Yasargil (Leyla) brain retractors were placed to maintain surgical access. Tumor had a thick purple capsule with adjacent yellowish gliotic tissue. Tumor capsule was friable and entered using suction and bipolar cautery. Hemorrhagic fluid content and blood clots mixed with necrotized tissue were suctioned. Tumor capsule was totally resected using suction and bipolar cautery under microscopic view till the gliotic tissue could be seen in entire periphery of surgical cavity. CSF egression from a small rent into right ventricle frontal horn was encountered during resection of deep medial parts of tumor capsule which was secured with a cotton patty to prevent blood backflow into ventricle. Meticulous hemostasis was achieved using oxidized regenerated cellulose and surgical site irrigation was done. Dura was sutured in a watertight fashion and bone flap was secured in place. Surgery duration was approximately two hours.

\section{Post-operative course:}

Patient was extubated immediately after emergence from anesthesia. Immediate post-op examination revealed complete consciousness without any significant changes in pre-operative motor status and with no surgically imposed new focal neurological deficit. Patient was admitted to pediatric ICU. On first postoperative day, there was considerable improvement in patient motor status with $3 / 5$ and $4 / 5$ muscle forces of left upper and lower extremities respectively. Agitation resolved and he had complete tolerance of oral feeding. Post-operative contrast enhanced MRI was performed on the day after surgery which demonstrated gross total resection with no signs of enhancing tumor or hemorrhage in the surgical cavity (Figure 3). After a 2-day care in pediatric ICU, patient passed another uneventful day in pediatric neurosurgery ward and was subsequently discharged from hospital with medication and physical therapy orders.

Patient was brought to us 4 days later with subgaleal CSF collection in the surgical site without any signs of infection or raised ICP. This was managed conservatively with intermittent application of compressive dressing at home and on subsequent follow-up visit 10 days later there were no signs of pseudomeningocele in place. Neurological status was significantly improved and patient was able to walk independently but there was still a $4 / 5$ paresis in left upper hand. After 4 months follow up, there is no sign of tumor recurrence and patient performs well without significant complaint or neurological deficit.

\section{Pathological findings:}

Histopathological examination revealed a moderately cellular mixed glioneuronal neoplasm in a myxoid bachground forming numerous perivascular pseudorosettes. Tumor cells were round and monotonous 
with low mitotic rate. Necrosis, atypia and endothelial proliferation were all absent (Figure 4). Immunohistochemistry (IHC) staining was positive for vimentin, S100, synaptophysin (scattered cells) and NSE and negative for EMA and CK. Also, $3 \%$ of cells were positive for Ki-67. These findings were consistent with PGNT (WHO grade I).

\section{Discussion}

\section{Epidemiology:}

PGNTs are newly described rare CNS tumors and the data about these tumors are limited to case reports and case series. PGNT is mostly seen in young adults with the reported mean age of 20-30 years in different case series[2,37]. But there are also limited reports of PGNTs in pediatric population or elderly. In our review of literature, we have found 41 cases of PGNTs reported in pediatric population with ages ranging from 3 to 17 years and mean age of 10.2 years. Among these 41 cases most of patients were in adolescence age followed by early and late childhood age groups (Figure 5). There is no PGNT reported in toddlers or infants and our report is the first in this regard. Sex preponderance is a controversial matter in these tumors with some case series reporting a slight male predilection and some others showing more tendency to females[2,37]. But overall, it seems that there is no significant sex preponderance in these tumors.

\section{Clinical features:}

Most of PGNTs are diagnosed following occurrence of neurological symptoms such as seizure, headache or nausea/vomiting. In a systematic review by Schlamann et al on 71 PGNT patients there was no reported patient with acute neurological deficit or loss of consciousness as presenting symptoms[37]. Subsequently, another larger systematic review performed by Ahmed et al confirmed these findings[2]. So, presenting symptoms of sudden onset hemiparesis and lowering of consciousness in our patient seems to be unique. Because these tumors are low grade slow growing tumors, the most anticipated clinical scenario is gradual progression of symptoms related to raised ICP or compressive effects of a space occupying lesion over time. But in our patient, there were no history of previous neurological symptomatology and presentation of symptoms was quite abrupt. This may be a consequence of patient young age which resulted in initial patient symptoms such as headache to remain latent.

\section{Imaging characteristics:}

PGNTs are mostly located in paraventricular areas of temporal and frontal lobes but there are reports of unusual locations such as cerebellum, pineal region or brainstem. Mean size of these tumors at diagnosis is reported as $4.3 \mathrm{~cm}[26,21,18]$. These tumors appear in MRI and CT scans as completely cystic, predominantly cystic with enhancing mural nodule, mixed cystic solid or rarely completely solid tumors. Perilesional edema is a common finding and areas of calcifications are not rare. These tumors show ring enhancement in MRI and CT after contrast administration. There are a few reports about PGNTs with histopathological or radiological evidence of chronic microhemorrhages AKA hemosiderosis[4,13,24] but 
intra-tumoral overt acute hemorrhage is extremely rare in these tumors with only one case reported up to now[10] which makes our case the second one.

\section{Histopathology:}

PGNTs are mixed tumors with both glial and neuronal components. The morphologic characteristics are pseudopapillae composed of hyalinized blood vessels with single or multiple lining layers of cuboidal cells. There is case to case difference in proportion of glial and neuronal elements[23,12]. However, these tumors may resemble ganglioglioma, pleomorphic xanthoastrocytoma, pilocytic astrocytoma and dysembryoplastic neuroepithelial tumor in histopathological examinations[4]. The reported average Ki-67 index of PGNTs is 3.6 with most cases having a Ki-67 index of $<5$ which is in agreement with our case (Ki67:3\%)[2].

\section{Management:}

Gross total resection (GTR) is the treatment of choice in these tumors with most of patients who received GTR having excellent prognosis[9,37]. Although these tumors are naturally amenable to GTR, but subtotal resection (STR) is possible due to intra-operative circumstances. Use of adjuvant chemotherapy or radiotherapy is rarely reported. In a meta-analysis on 71 patients with PGNT, $6 \%$ and $10 \%$ of patients had received chemotherapy and radiotherapy respectively mostly after STR[37]. In another meta-analysis, tumors with higher Ki-67 index were more likely to be treated with radiation therapy[2]. Possible indications of adjuvant therapy can be high proliferation index, inoperability and recurrent disease[13,40]. Regular imaging surveillance help to detect any tumor recurrence and repeat surgical resection is recommended in these cases[37].

\section{Outcome:}

Ahmed et al in review of 138 patients with PGNT found that GTR has the advantage of increased 2-year progression free survival (PFS) over STR. Also, high Ki-67 index was associated with lower 5-year PFS. In their meta-analysis, survival was not influenced by patient age but male gender was predictive of higher 5-year PFS[2]. In another similar but smaller meta-analysis, Schlamann and colleagues showed that gender or age has no significant association with PFS. But survival analyses identified the Ki67 index and maximum tumor diameter as significant predictive factors for PFS with a 1\% increase in Ki-67 index increasing tumor progression by $10 \%$. This review measured 1.5-year PFS and overall survival (OS) of PGNTs as $86 \%$ and $98 \%$ respectively[37].

\section{Conclusion}

Our report demonstrates that PGNT although rare, can be seen in very young children and this diagnosis should be considered as last differential diagnoses even in toddlers. Also, it should be kept in mind that these tumors have potential for overt intra-tumoral hemorrhage and acute presentation with focal 
neurological deficits mimicking more common pathologies which should be considered to plan optimal patient management.

\section{Declarations}

Disclosure of interest: The authors declare that they have no known competing financial interests or personal relationships that could have appeared to influence the work reported in this paper.

Informed consent was obtained from patient parents as his legal guardians for publishing his data. However, we did not include any information which reveals patient identity in this manuscript. According to our regional ethics committee regulations, ethics approval was not needed for case reports which do not reveal patient identity

\section{References}

1. Agarwal S, Sharma MC, Singh G, Suri V, Sarkar C, Garg A, Kumar R, Chandra PS (2012) Papillary glioneuronal tumor-a rare entity: report of four cases and brief review of literature. Child's Nervous System 28:1897-1904

2. Ahmed AK, Dawood HY, Gerard J, Smith TR (2017) Surgical Resection and Cellular Proliferation Index Predict Prognosis for Patients with Papillary Glioneuronal Tumor: Systematic Review and Pooled Analysis. World Neurosurg 107:534-541. doi:10.1016/j.wneu.2017.08.041

3. Atri S, Sharma MC, Sarkar C, Garg A, Suri A (2007) Papillary glioneuronal tumour: a report of a rare case and review of literature. Child's Nervous System 23:349-353

4. Barnes NP, Pollock JR, Harding B, Hayward RD (2002) Papillary glioneuronal tumour in a 4-year-old. Pediatric neurosurgery 36:266-270

5. Benzagmout M, Karachi C, Mokhtari K, Capelle L (2013) Hemorrhagic papillary glioneuronal tumor mimicking cavernoma: two case reports. Clinical neurology and neurosurgery (Dutch-Flemish ed) 115:200-203

6. Bisson EF, Pendlebury WW, Horgan MA (2005) Glioneuronal tumor with unique imaging and histologic features. J Neurooncol 72:89-90. doi:10.1007/s11060-004-2277-y

7. Borges G, Bonilha L, Menezes AS, Queiroz LdS, Carelli EF, Zanardi V, Menezes JR (2004) Long term follow-up in a patient with papillary glioneuronal tumor. Arquivos de neuro-psiquiatria 62:869-872

8. Bridge JA, Liu Xq, Sumegi J, Nelson M, Reyes C, Bruch LA, Rosenblum M, Puccioni MJ, Bowdino BS, McComb RD (2013) Identification of a novel, recurrent SLC44A1-PRKCA fusion in papillary glioneuronal tumor. Brain pathology 23:121-128

9. Broholm H, Madsen F, Wagner A, Laursen H (2002) Papillary glioneuronal tumor-a new tumor entity. Clinical neuropathology 21:1-4

10. Buccoliero AM, Giordano F, Mussa F, Taddei A, Genitori L, Taddei GL (2006) Papillary glioneuronal tumor radiologically mimicking a cavernous hemangioma with hemorrhagic onset. Neuropathology 


\section{6:206-211}

11. Castro Castro J, Lista Martinez O, Carames Diaz N, Conde Lorenzo N (2019) Papillary glioneuronal tumor. A case report. Neurocirugia (Astur) 30:144-148. doi:10.1016/j.neucir.2018.04.003

12. Doxtader EE, Sturgis CD (2018) Cytopathologic features of papillary glioneuronal tumor. Diagn Cytopathol 46:284-286. doi:10.1002/dc.23885

13. Epelbaum S, Kujas M, Van Effenterre R, Poirier J (2006) Two cases of papillary glioneuronal tumours. British journal of neurosurgery 20:90-93

14. Faria C, Miguéns J, Antunes JL, Barroso C, Pimentel J, Martins MDC, Moura-Nunes V, Roque L (2008) Genetic alterations in a papillary glioneuronal tumor: Case report. Journal of Neurosurgery: Pediatrics 1:99-102

15. Fujita Y, Kinoshita M, Ozaki T, Kitamura M, Nakatsuka SI, Kanemura Y, Kishima H (2018) Enlargement of papillary glioneuronal tumor in an adult after a follow-up period of 10 years: a case report. J Surg Case Rep 2018:rjy123. doi:10.1093/jscr/rjy123

16. Gelpi E, Preusser M, Czech T, Slavc I, Prayer D, Budka H (2007) Papillary glioneuronal tumor. Neuropathology 27:468-473

17. Goethe EA, Youssef M, Patel AJ, Jalali A, Goodman JC, Mandel JJ (2019) Recurrent Papillary Glioneuronal Tumor. World Neurosurg 128:127-130. doi:10.1016/j.wneu.2019.04.266

18. Husain N, Husain M (2009) Endoscopic diagnosis of a pineal papillary glioneuronal tumor with extensive ventricular involvement: case report with review of literature. Neurology India 57:792

19. Iżycka-Świeszewska E, Majewska H, Szurowska E, Mazurkiewicz-Bełdzińska M, Drożyńska E (2008) Papillary glioneuronal tumour of the precentral gyrus. Folia neuropathologica 46:158-163

20. Javahery RJ, Davidson L, Fangusaro J, Finlay JL, Gonzalez-Gomez I, McComb JG (2009) Aggressive variant of a papillary glioneuronal tumor: report of 2 cases. Journal of Neurosurgery: Pediatrics 3:4652

21. Kaloostian PE, Chen H, Tran HP (2013) Malignant papillary glioneuronal tumor of the pineal gland: case presentation and literature review of a distinct entity. The American journal of case reports $14: 164$

22. Kleihues P, Louis DN, Scheithauer BW, Rorke LB, Reifenberger G, Burger PC, Cavenee WK (2002) The WHO classification of tumors of the nervous system. J Neuropathol Exp Neurol 61:215-225; discussion 226-219. doi:10.1093/jnen/61.3.215

23. Komori T, Scheithauer BW, Anthony DC, Rosenblum MK, McLendon RE, Scott RM, Okazaki H, Kobayashi M (1998) Papillary glioneuronal tumor: a new variant of mixed neuronal-glial neoplasm. The American journal of surgical pathology 22:1171-1183

24. Konya D, Peker S, Ozgen S, Kurtkaya O, Pamir MN (2006) Superficial siderosis due to papillary glioneuronal tumor. Journal of clinical neuroscience 13:950-952

25. Kordek R, Hennig R, Jacobsen E, Kearney M (2003) Papillary glioneuronal tumor-a new variant of benign mixed brain neoplasm. Pol J Pathol 54:75-78 
26. Li D, Wang J-M, Li G-L, Hao S-Y, Yang Y, Wu Z, Zhang L-W, Zhang J-T (2014) Clinical, radiological, and pathological features of 16 papillary glioneuronal tumors. Acta neurochirurgica 156:627-639

27. Louis DN, Ohgaki H, Wiestler OD, Cavenee WK, Burger PC, Jouvet A, Scheithauer BW, Kleihues $P$ (2007) The 2007 WHO classification of tumours of the central nervous system. Acta Neuropathol 114:97-109. doi:10.1007/s00401-007-0243-4

28. Mahajan H, Varikatt W, Dexter M, Boadle R, $\mathrm{Ng} \mathrm{T}$ (2010) Papillary glioneuronal tumor of the frontal lobe. Journal of Clinical Neuroscience 17:534-536

29. Momota H, Fujii M, Tatematsu A, Shimoyama Y, Tsujiuchi T, Ohno M, Natsume A, Wakabayashi T (2014) Papillary glioneuronal tumor with a high proliferative component and minigemistocytes in a child. Neuropathology 34:484-490

30. Myung JK, Byeon S-j, Kim B, Suh J, Kim S-K, Park C-K, Chung CK, Chang K-H, Park S-H (2011) Papillary glioneuronal tumors: a review of clinicopathologic and molecular genetic studies. The American journal of surgical pathology 35:1794-1805

31. Page P, Meylor J, Li Y, Patel V, Ahmed A (2019) Tectal region papillary neuroglial tumour: a case report. Br J Neurosurg:1-3. doi:10.1080/02688697.2018.1562035

32. Pages M, Lacroix L, Tauziede-Espariat A, Castel D, Daudigeos-Dubus E, Ridola V, Gilles S, Fina F, Andreiuolo F, Polivka M (2015) Papillary glioneuronal tumors: histological and molecular characteristics and diagnostic value of SLC44A1-PRKCA fusion. Acta neuropathologica communications 3:85

33. Phi JH, Park S-H, Chae JH, Wang K-C, Cho B-K, Kim S-K (2010) Papillary glioneuronal tumor present in a patient with encephalocraniocutaneous lipomatosis: case report. Neurosurgery 67:E1165-E1169

34. Pimentel J, Barroso C, Miguens J, Firmo C, Antunes J (2009) Papillary glioneuronal tumor-prognostic value of the extension of surgical resection. Clinical neuropathology 28:287-294

35. Portela-Oliveira E, Torres US, Lancellotti CLP, Souza AS, Ferraz-Filho JRL (2014) Solid intraventricular papillary glioneuronal tumor: magnetic resonance imaging findings with histopathological correlation. Pediatric neurology 50:199-200

36. Rocka S, Neverauskiene L, Nelson EL, Burneikiene S (2019) Papillary Glioneuronal Tumour: A Case Report. Cureus 11:e4215. doi:10.7759/cureus.4215

37. Schlamann A, von Bueren AO, Hagel C, Zwiener I, Seidel C, Kortmann RD, Muller K (2014) An individual patient data meta-analysis on characteristics and outcome of patients with papillary glioneuronal tumor, rosette glioneuronal tumor with neuropil-like islands and rosette forming glioneuronal tumor of the fourth ventricle. PLoS One 9:e101211. doi:10.1371/journal.pone.0101211

38. Stosic-Opincal T, Peric V, Gavrilovic S, Gavrilov M, Markovic Z, Sener R (2005) Papillary glioneuronal tumor. American Journal of Roentgenology 185:265-267

39. Vajtai I, Kappeler A, Lukes A, Arnold M, Lüthy AR, Leibundgut K (2006) Papillary glioneuronal tumor. Pathology-Research and Practice 202:107-112

40. Vaquero J, Coca S (2007) Atypical papillary glioneuronal tumor. Journal of neuro-oncology 83:319323 
41. Wang DD, Piao YS, Blumcke I, Sun YN, Gao W, Zhao LH, Wang WM, Lu DH (2018) Papillary Glioneuronal Tumor with an Excessive Angiomatous Component in an Elderly Man. Chin Med J (Engl) 131:243-244. doi:10.4103/0366-6999.222333

42. Xiao H, Ma L, Lou X, Gui Q (2011) Papillary glioneuronal tumor: radiological evidence of a newly established tumor entity. Journal of Neuroimaging 21:297-302

43. Yadav N, Rao S, Saini J, Prasad C, Mahadevan A, Sadashiva N (2017) Papillary glioneuronal tumors: A radiopathologic correlation. Eur J Radiol 97:44-52. doi:10.1016/j.ejrad.2017.10.004

44. Zhao R-J, Zhang X-L, Chu S-G, Zhang M, Kong L-F, Wang Y (2016) Clinicopathologic and neuroradiologic studies of papillary glioneuronal tumors. Acta neurochirurgica 158:695-702

\section{Figures}
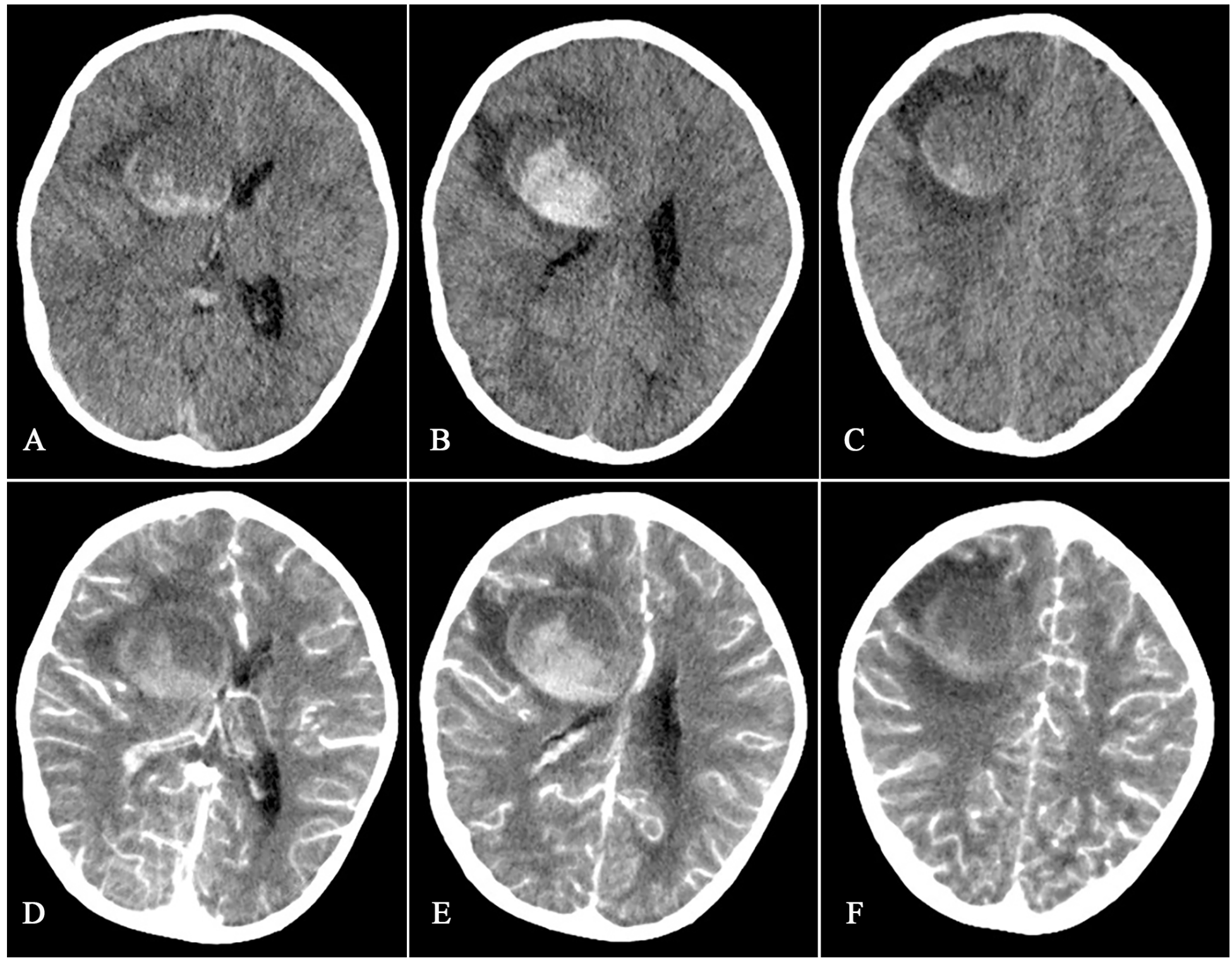

Figure 1 
Pre-operative brain CT scan of the patient showing a round, well defined heterogenous lesion located adjacent to frontal horn of right lateral ventricle extending superiorly toward centrum semiovale. Axial non-contrast images show intra-tumoral hemorrhage with compressive effect on lateral ventricle and subsequent midline shift $(A, B, C)$. Rim enhancement and lack of any significant vascular origin can be seen after contrast administration (D,E,F)

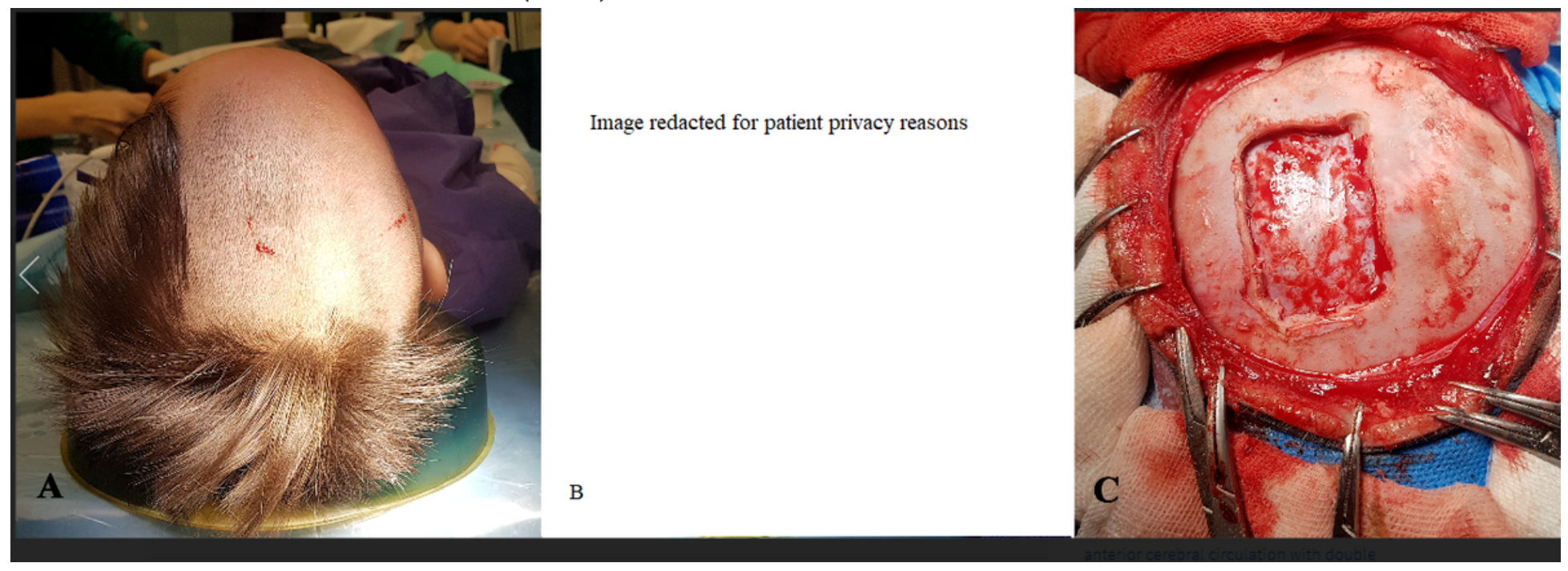

\section{Figure 2}

Intra-operative photograph of patient positioning $(A, B)$ and surgical view of small frontal craniotomy used for trans-frontal transcortical approach to tumor (C) 


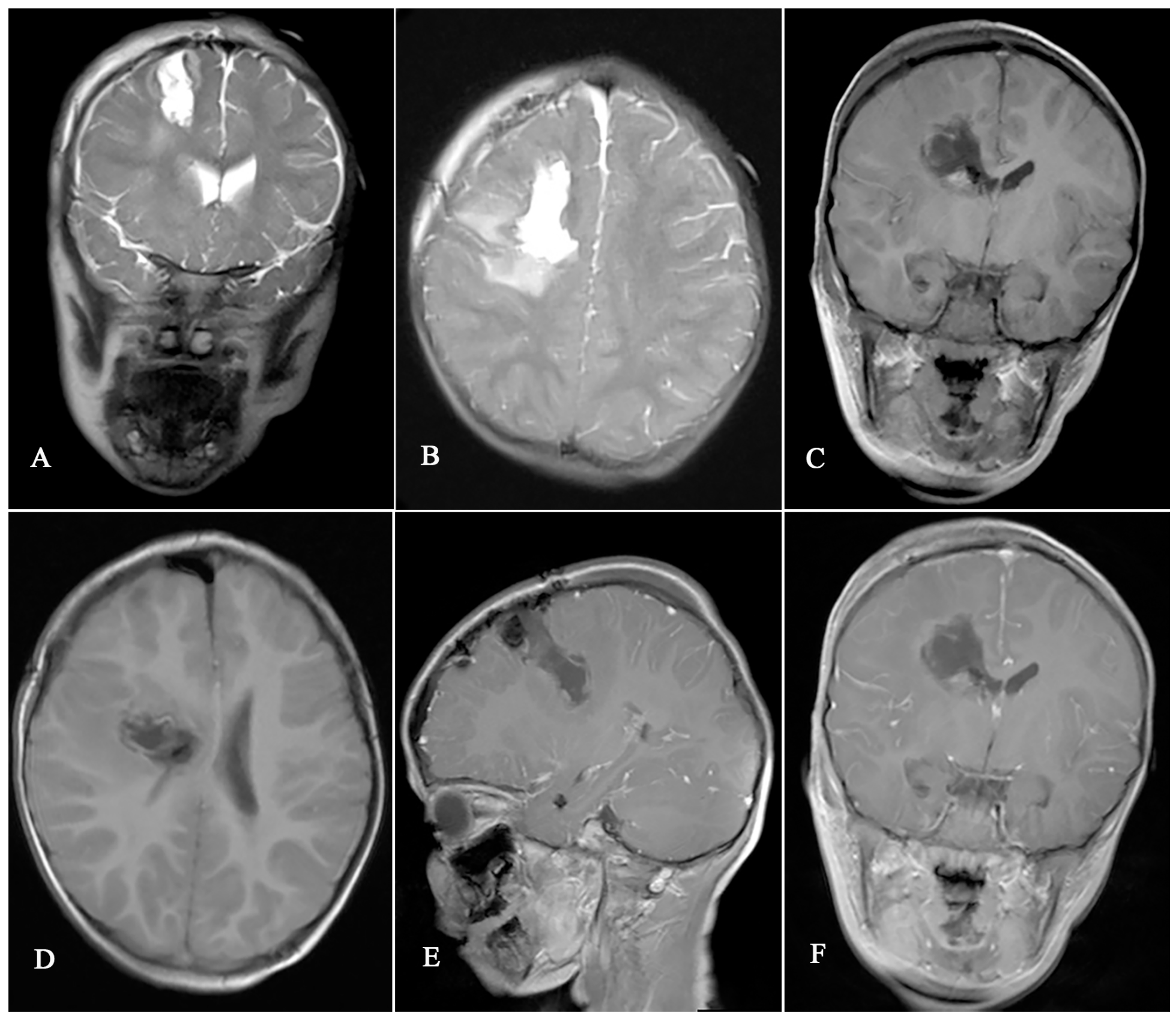

Figure 3

Post-operative MRI showing achievement of GTR. A and B depict safe entry point in right middle frontal gyrus and medial-posterior trajectory of surgical corridor used for tumor resection. Coronal non-contrast (C) and axial (D), sagittal (E) and coronal (F) contrast enhanced images showing no significant residual enhancement confirming GTR 


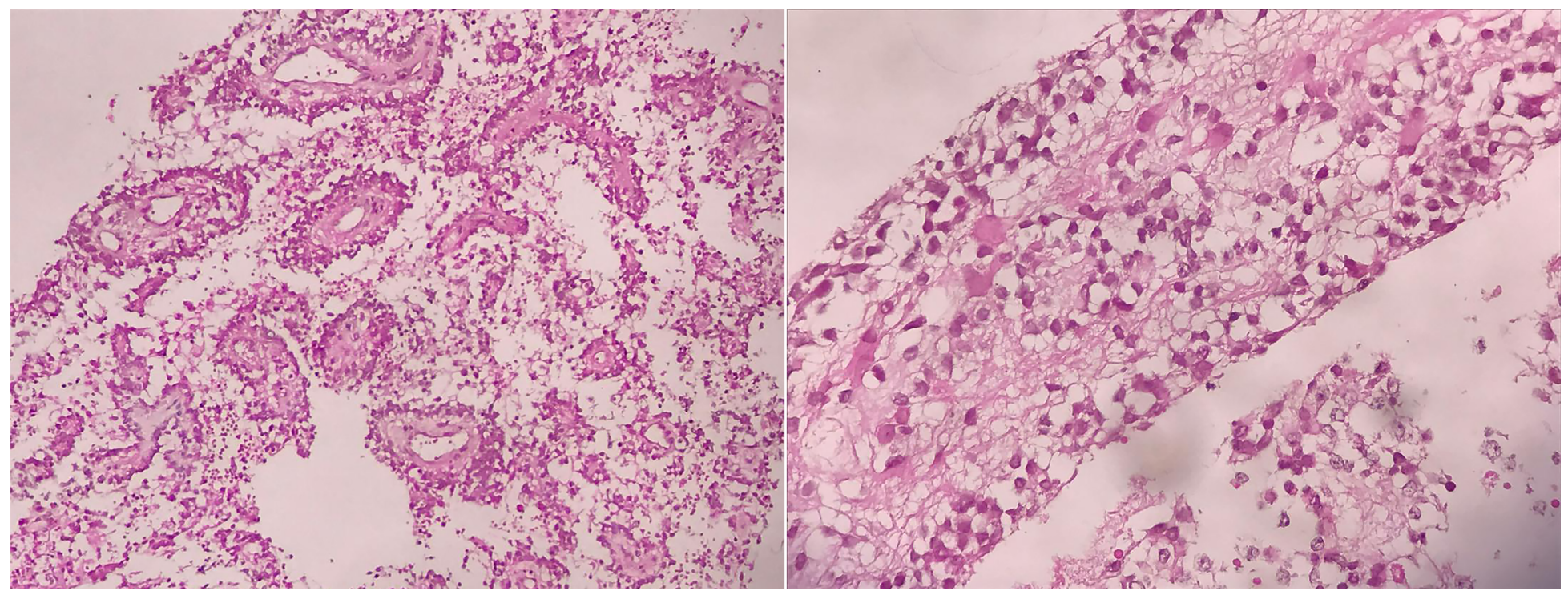

\section{Figure 4}

Histopathological examination of resected tumor. Left, low power field image (10x magnification) shows pseudopapillary structures in a loose myxoid background. Right, high power field image (40x magnification) showing neuronal compartment of tumor without any signs of malignant characteristics. Both images are with H\&E staining

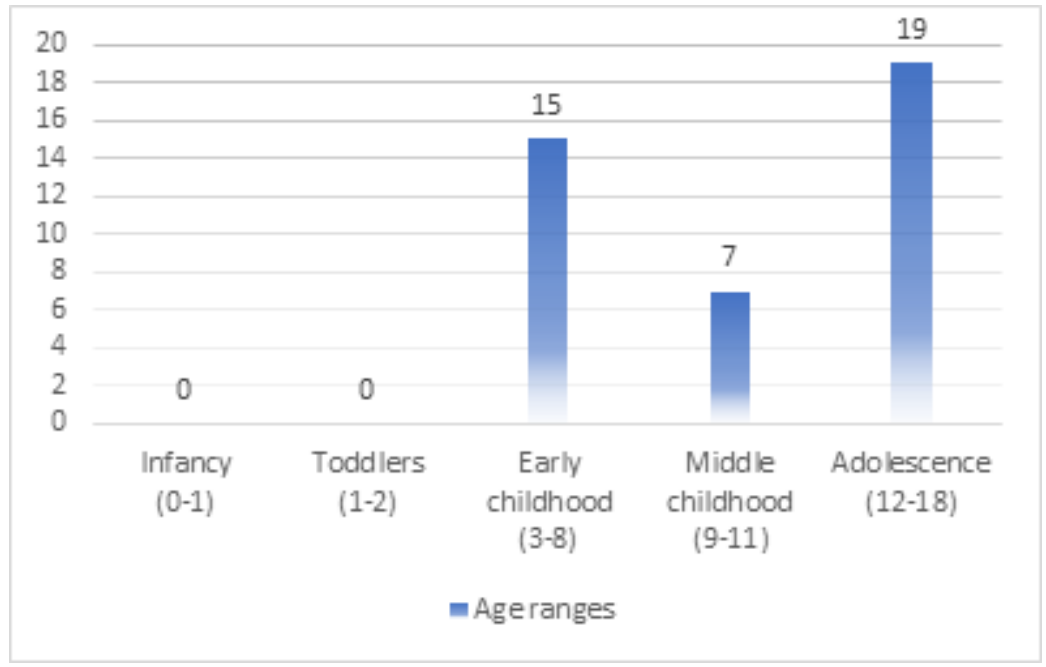

\section{Figure 5}

Age distribution of 41 cases of pediatric PGNT reported in the literature which depicts no available report of PGNT in toddlers or infants till this report 